\title{
Tomografía Computarizada en Bajas Latitudes de la lonósfera: Avances Recientes
}

\author{
Melissa Medrano ${ }^{1}$, Meyer Merino ${ }^{1}$, Juan Pablo Velásquez², Marcos Inoñan ${ }^{3}$, Enrique Rojas ${ }^{4}$ \\ ${ }^{1}$ Universidad Peruana de Ciencias Aplicadas, Lima, Perú \\ ${ }^{2}$ Radio Observatorio de Jicamarca, Lima, Perú \\ ${ }^{3}$ Washington University, Washington, EE.UU \\ ${ }^{4}$ Cornell University, Nueva York, EE.UU
}

Recibido el 23 de diciembre del 2017, aceptado el 30 de diciembre del 2017

DOI: https://doi.org/10.33017/RevECIPeru2017.0011/

\section{Resumen}

La distribución de la densidad de electrones es, probablemente, el parámetro físico más relevante al momento de estudiar la electrodinámica del espacio cercano a la tierra. Existen diversas técnicas de la teledetección, y alta precisión, que tienen como objetivo estimar su distribución. Sin embargo, la gran mayoría de estas se encuentran limitadas a reconstrucciones de una sola dimensión. En contraste, la tomografía de la ionósfera surge como la alternativa más prometedora para producir perfiles 2D o 3D. Utiliza señales satelitales, que viajan a lo largo de un plano de reconstrucción definido, para construir un modelo lineal basado en la relación teórica entre las fases de estas señales y la densidad de electrones del plasma ionosférico. La solución a este sistema proporciona una estimación de la distribución de densidad de electrones. El uso de esta técnica tiene como objetivo reconstruir los perfiles de densidad, tanto para los estados estables de la ionosfera, como para los estados en los que la ionosfera contiene irregularidades en el plasma.

Descriptores: lonósfera, ciencias espaciales, física de plasma, computación científica, tomografía, radio definida por software.

\section{Abstract}

The electron density distribution is, arguably, the most relevant physical parameter to study the geospace electrodynamics. There are several high precision remote sensing techniques to estimate this distribution, unfortunately, most of them are constrained to a one-dimensional reconstruction. In contrast, lonospheric tomography is probably the most promising alternative to produce $2 \mathrm{D}$ or $3 \mathrm{D}$ profiles. It uses satellites signals, that travel along a defined reconstruction plane, to construct a linear model based on the theoretical relation between this signals phases and the electron density of ionospheric plasma. The solution of this system provides an estimation of the electron density distribution. The use this technique aims to reconstruct density profiles, both, for stable states of the ionosphere and states in which the ionosphere contains plasma irregularities. In

Keywords: lonosphere, space science, plasma physics, scientific computing, tomography, software-defined radio.

\section{Introducción}

Actualmente, existen varias técnicas experimentales para estimar parámetros físicos del plasma en la ionósfera. Aunque algunos de ellos son muy precisos, como Incoherent Scatter Radar
(ISR) o sistemas de medición montados en cohetes. Sin embargo, la gran mayoría de estas se encuentran limitadas a mediciones de trayectorias unidimensionales. Otras técnicas, como Coherent Radar, comúnmente se basan en relaciones empíricas específicas para estimar parámetros 
fundamentales como la densidad de electrones $\left(n_{e}\right)$, la cual es, discutiblemente, la variable más importante en la dinámica del plasma.

La Tomografía Computarizada de la lonósfera, CIT por sus siglas en inglés, fue propuesta por primera vez por Austen [1] para obtener perfiles de densidad en dos dimensiones con la información del Contenido Total de Electrones, TEC por sus siglas en inglés, provisto por los receptores. Esta técnica nos permite estudiar estructuras de plasma importantes, como las burbujas de plasma y su dinámica espacial.

Después del artículo de Austen, muchos otros enfoques han sido propuestos. Para empezar, la mayoría de reconstrucciones de CIT fueron desarrolladas usando métodos de reconstrucción algebraicos, pero con el paso de algunos años, otros enfoques fueron usados, como optimización regularizada o estimación bayesiana [2]. El diseño experimental también varió, primero pensado para satélites de baja órbita (Low Earth Orbit (LEO) satellites), CIT ha sido aplicada a data de TEC obtenida mediante el uso de GPS con receptores en tierra y a través de experimentos de Radio Occultation [2].

Este proyecto busca probar nuevos enfoques para la reconstrucción utilizando CIT con el objetivo de estudiar el principal mecanismo causante de irregularidades de escala kilométrica alrededor de la ionósfera ecuatorial denominado "Spread F". De esta forma, se busca entender la dinámica espacial de estas estructuras, fundamental para las ciencias espaciales, no solo debido a la gran cantidad de conexiones causales con otros fenómenos, sino también porque suelen afectar el flujo de información de los sistemas de navegación satelital. La distribución del trabajo comenzará con la sección 2 , donde se proporcionará un breve resumen del progreso y la perspectiva de los pasos futuros. Asimismo, contendrá la definición del problema en términos matemáticos y las aproximaciones numéricas involucradas. Luego, en la sección 3 se presentarán los resultados con métodos de inversión utilizados, hasta el momento, el análisis de los resultados y otros métodos que se están evaluando usar. En la sección 4 se dará un breve resumen de la determinación de los planos de reconstrucción, que implica, además, el diseño del sistema de recepción. Finalmente, las conclusiones son presentadas en la sección 5 .

\section{Metodología}

\subsection{Simulación de la ionósfera referencial y los datos de la ionosonda}

Hasta el momento se han llevado a cabo reconstrucciones tomográficas a partir de simulaciones de la ionósfera utilizando el modelo de Chapman [4] más el agotamiento de la densidad de electrones $n_{e}$ modelado con funciones gaussianas. Los perfiles de densidad producidos a partir de este modelo son muy simples, por lo que hemos empezado a probar otros modelos $y / 0$ perfiles ionosféricos a partir de bases de datos de diferentes instrumentos de medición. Un ejemplo es el proyecto IRI (International Reference lonosphere) que produce modelos estándares empíricos basados en bases de datos de una red mundial de ionosondas, radares de dispersión incoherentes de mucha potencia, por ejemplo, los del Radio Observatorio de Jicamarca (ROJ), entre otros.

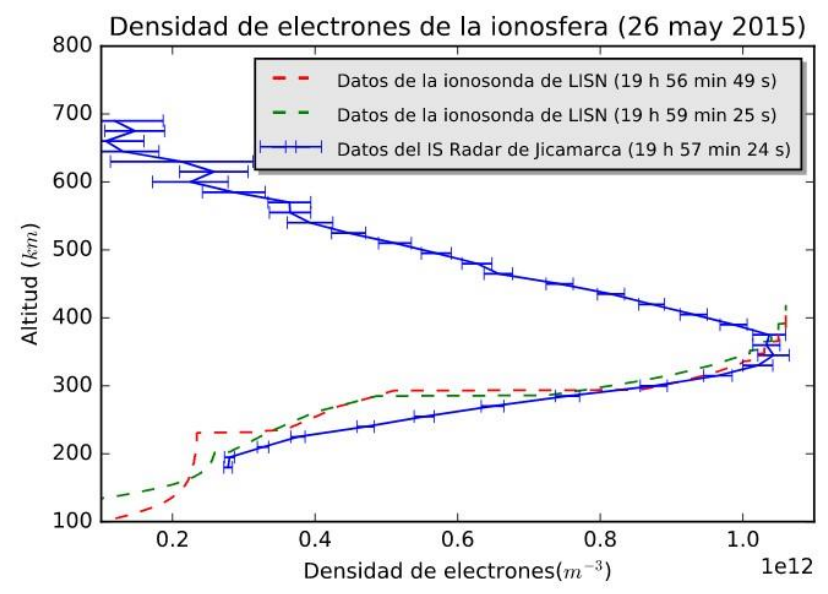

Figura 1: Comparación de datos de $n_{e}$ entre LISN y Madrigal.

Por otro lado, Low-Latitude Ionospheric Sensor Network (LISN) es un proyecto internacional cuya finalidad es estudiar y pronosticar los fenómenos ionosféricos, y consiste en un sistema capaz de monitorear la atmósfera a través de modernos instrumentos geofísicos, como las ionosondas, que entre otras cosas proporcionan información sobre alturas y estimaciones de perfiles de densidades de la parte inferior de la ionósfera.

En ese sentido, estamos en proceso de analizar los perfiles de densidad de electrones en función de la 
altitud para comparar y complementar dicha información con la del experimento en modo "Jicamarca Oblique" de los radares de dispersión

incoherente de Jicamarca de la base de datos de Madrigal.

Dicha base de datos es capaz de administrar y servir datos de archivos en tiempo real, en una gran variedad de formatos, y desde una amplia gama de instrumentos científicos de la atmósfera superior; véase Figura 1.

\subsection{Discretización de la ionósfera}

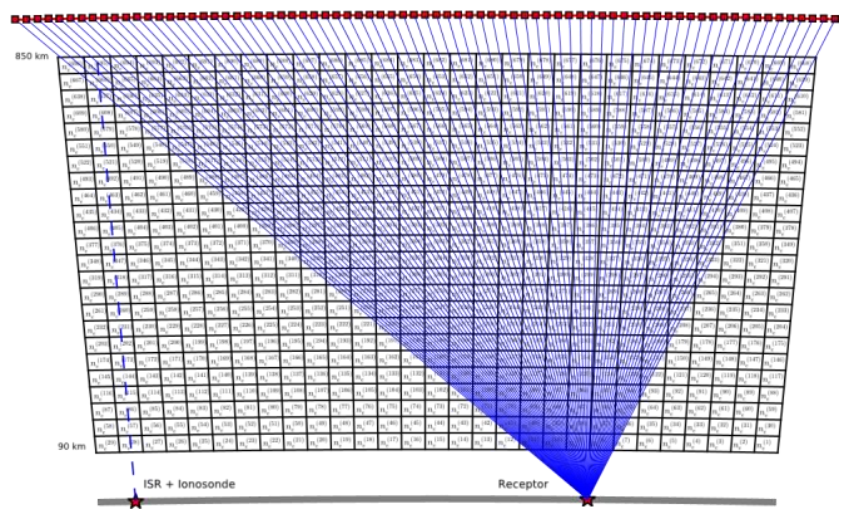

Figura 2: llustración de la ionósfera discretizada y las trayectorias $S R$.

La discretización de la ionósfera es crucial para la estimación de los perfiles de densidad de electrones. Se toman cortes aproximadamente bidimensionales, ya que se cubre una zona de longitud constante, y se discretiza en celdas de altitud y latitud, con dimensiones tales que la densidad de electrones en cada celda puede considerarse constante.

Sea $f$ la frecuencia de la señal emitida por el satélite, tal que cumpla la condición $f \gg f_{p}$, donde $f_{p}$ es la frecuencia del plasma, podemos expresar el TEC, en la trayectoria Satélite-Receptor (SR), mediante la siguiente relación [4]:

$$
\mathrm{TEC}=\int_{P} n_{e} d \boldsymbol{l}
$$

Donde $P$ es la trayectoria SR y $n_{e}$ la densidad de electrones a lo largo de dicha trayectoria. Usando las mismas asunciones, podemos medir el TEC según:

$$
\mathrm{TEC} \approx c \frac{f_{1}^{2} f_{2}^{2} \Delta \tau}{40,3\left(f_{1}^{2}-f_{2}^{2}\right)}
$$

Donde $f_{1}$ y $f_{2}$ son las frecuencias de las dos señales emitidas por el satélite al receptor, y $\Delta \tau$ es la diferencia de tiempo de recepción entre las dos

señales. Estas integrales de línea están contenidas en un plano de reconstrucción como el mostrado en la Figura 2. Como consecuencia de un proceso de discretización adecuado, la integral de línea en ec.(1) se puede expresarse como la siguiente sumatoria:

$$
\int_{P} n_{e} d \boldsymbol{l} \approx \sum_{i} n_{e}^{(i)} \Delta l_{i}=\boldsymbol{B} . \boldsymbol{n}_{e}
$$

Donde $\quad \boldsymbol{B}=(0, \ldots, 0, \Delta l 1,0, \ldots, 0, \Delta l 2,0, \ldots) . \quad$ La posición y el valor de $\Delta l_{i}$ están determinados por la celda atravesada por la trayectoria $S R$ y por el tamaño del segmento contenido en esta. Un conjunto de mediciones de TEC, $b$, tomado para diferentes trayectorias SR puede ser relacionado a un conjunto de valores de $n_{e}$ en el grid mediante el siguiente sistema lineal:

$$
\hat{A} . \boldsymbol{n}_{e}=\boldsymbol{b}
$$

Donde $\hat{A}$, la matriz base, contiene la información de las posiciones y tamaños de los segmentos en cada celda del grid correspondiente a múltiples trayectorias SR que atraviesan el grid.

$$
\left[\begin{array}{ccc}
a_{11} & \cdots & a_{1 N} \\
\vdots & \ddots & \vdots \\
a_{m 1} & \cdots & a_{m N}
\end{array}\right]_{m \times N}\left[\begin{array}{c}
n_{e}^{(1)} \\
\cdots \\
n_{e}^{(N)}
\end{array}\right]_{N}=\left[\begin{array}{c}
\mathrm{TEC}_{1} \\
\cdots \\
\mathrm{TEC}_{N}
\end{array}\right]_{m}
$$

Una matriz binaria fue utilizada para estudios preliminares. Esta matriz tenía como elementos 1 ó 0 , dependiendo de si una trayectoria SR pasaba por una celda o no. Los resultados mostrados ahora fueron reproducidos con la matriz $\widehat{M}$ definida en esta sección.

\section{Resultados}

\subsection{Técnicas de inversión}

La mayoría de problemas en tomografía se reducen a resolver sistemas de ecuaciones lineales, así que la elección de adecuados algoritmos de reconstrucción es de crucial importancia para obtener los mejores resultados posibles en este tipo de problemas [4].

El sistema construido en ec. (4) ha sido resuelto mediante el uso de los algoritmos ART, SIRT y MART [5]. Estos métodos iterativos son 
relativamente sencillos de implementar. Dado el hecho de que ninguna matriz completa será almacenada, el uso de memoria es mínimo.

Trabajos previos encontraron que estos algoritmos son eficientes al lidiar con sistemas lineales sobredeterminados y sub-determinados al mismo tiempo (ill-posed systems) [3]. Las expresiones iterativas de las que se valen los dos algoritmos mencionados son las siguientes:

MART

$$
n_{e j}^{(k+1)}=n_{e j}^{(k)} \times\left(\frac{b_{i}}{\left\langle\hat{A}^{i}, n_{e}^{(k)}\right\rangle}\right)^{\frac{\lambda_{k} A_{j}^{i}}{\left|\hat{A}^{i}, \hat{A}^{i}\right\rangle}}, j=1, \ldots, N
$$

SIRT

$$
n_{e j}^{(k+1)}=n_{e j}^{(k)}+\frac{1}{P_{j}} \sum_{i} \lambda_{k} \frac{b_{i}-\left\langle\hat{A}^{i}, \boldsymbol{n}_{e}^{(k)}\right\rangle}{\left\langle\hat{A}^{i}, \hat{A}^{i}\right\rangle}, j=1, \ldots, N
$$

$\lambda_{j}$ es un factor de convergencia y $P_{j}$, el número de elementos diferentes de cero en la $j$-ésima columna de la matriz $\hat{A}$. El algoritmo MART converge a la solución de máxima entropía, mientras que SIRT minimiza $\left\|\hat{A} \boldsymbol{n}_{e}-b\right\|$ mediante la proyección de la nueva solución estimada en cada hiperplano definido por las filas $\widehat{A}^{\natural}$. El término de corrección en SIRT es calculado después de que todas las filas son proyectadas, para obtener el promedio de la contribución de todos los rayos atravesando la $j-$ ésima celda del grid.

Dada la naturaleza de estos algoritmos, se espera que las soluciones dadas por SIRT sean más suaves y menos sensibles a errores, mientras que MART es mejor para resolver estructuras pequeñas.

Los resultados preliminares de nuestras estimaciones pueden resumirse en la Figura 3, que muestra reconstrucciones obtenidas con una versión simplificada de la ec.(3), en donde se asumió que cada $\Delta l_{i}=1$ y se utilizaron curvas de Chapman para inicializar la simulación y determinar la ionósfera modelo. A pesar de estas simplificaciones, la Figura 3 muestra las principales características de los métodos SIRT y MART.

Como se explicó en la primera sección, hemos podido mejorar nuestras simulaciones. La figura 4 , muestra la nueva ionósfera modelo, creada con estimaciones del modelo IRI para simular el estado estable $\left(n_{0}\right)$ y gaussianas ellipticas para modelar burbujas del plasma $(\delta n)$, característicos del "Spread F".
Con estas definiciones, podemos decir que la ionósfera modelo será representada por $n_{e}=$ $n_{0}(1+\delta n)$. La mejora en la discretización por otro

lado, permite representar la integral de línea en la ec.(1) de manera mucho más precisa.

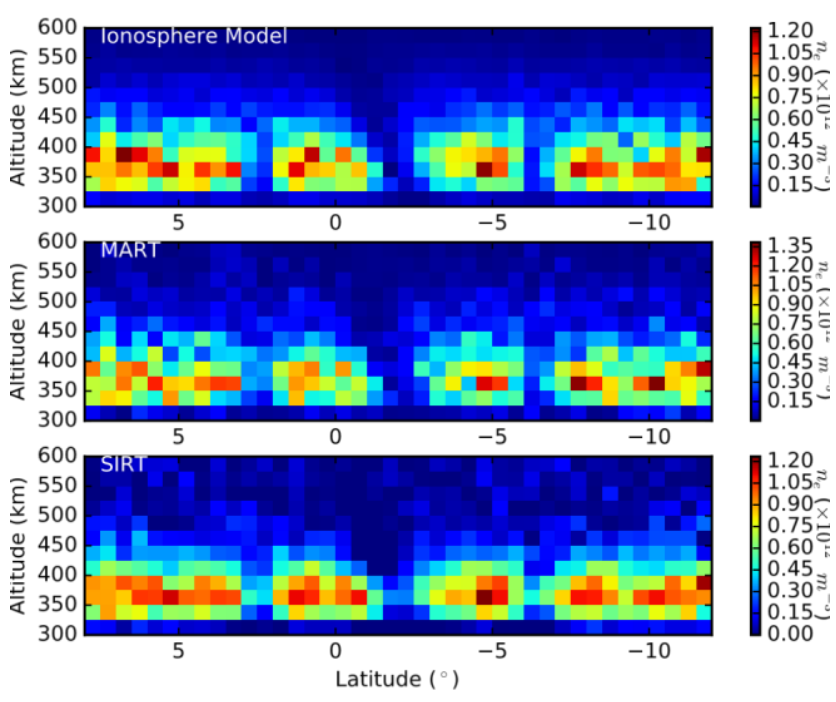

Figura 3: Inversiones con 5 receptores y 100 posiciones de satélite.

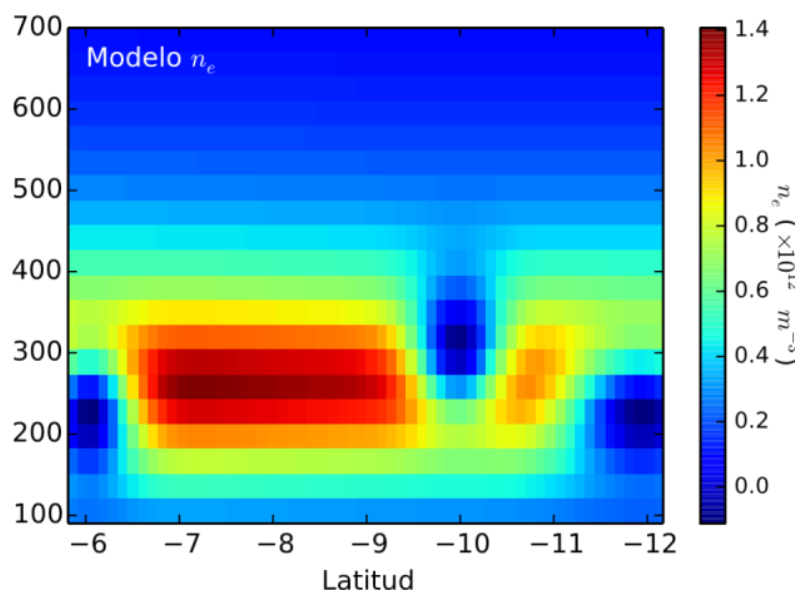

Figura 4: Inversiones con 5 receptores y 100 posiciones de satélite.

La matrix base $\widehat{M}$ asociada a esta nueva discretización, para el caso de una malla de $20 \times 60$, para acelerar la inversión se deberían implementar 4 receptores y 50 posiciones del satélite se puede apreciar en la Figura 5. Donde vemos que $\widehat{M}$ es sumamante "sparse", lo que sugiere que métodos numéricos que contemplen matrices de este tipo.

Los datos simulados de TEC se obtienen mediante $b=n_{e}+\varepsilon$, donde $b$ contiene los valores simulados de TEC y $\varepsilon$ es un error asociado a la 
Las características de un receptor definen la calidad de la señal medida y su selección resulta un punto

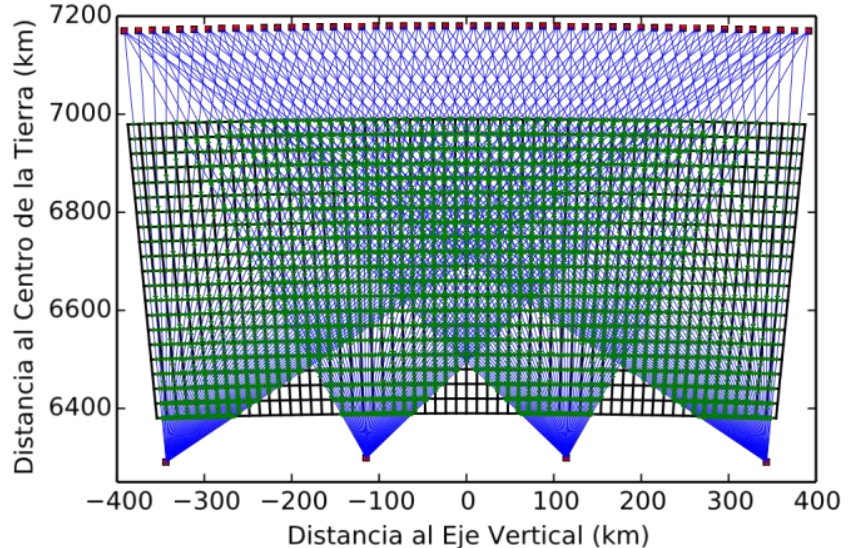
Matriz Base $\hat{M}$

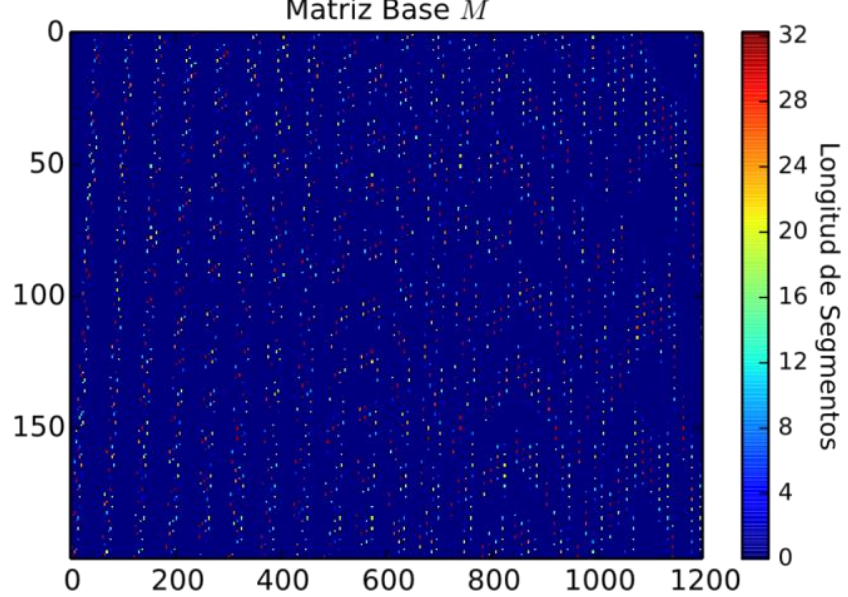

Figura 5: (Arriba) Malla de 20 × 60 y 200 mediciones de TEC. (Abajo) Matriz base $M^{\wedge}$ asociada a la geometría de del problema.

Se pueden apreciar claramente 4 curvas, éstas corresponden a los 4 receptores. En un escenario real, este sería el tipo de información que tendríamos para realizar las reconstrucciones.

Aplicando los algoritmos de ART y MART podemos obtener de los datos simulados de TEC, un perfil de densidad electrónica $n_{e}$, Figura 7 . Las reconstrucciones de la Figura 7 se realizaron con 15 iteraciones sobre todas las filas de la matriz $\widehat{M}$. La reconstrucción con MART logró tener un error relativo a la ionósfera modelo 5 veces menor que la realizada con ART. Además, podemos ver claramente que MART reproduce mejor las características principales de las irregularidades y las gradientes.

\section{Sistema de recepción}

\subsection{Diseño de un receptor de bajo costo}

clave en la elaboración de cualquier estudio de la ionósfera.

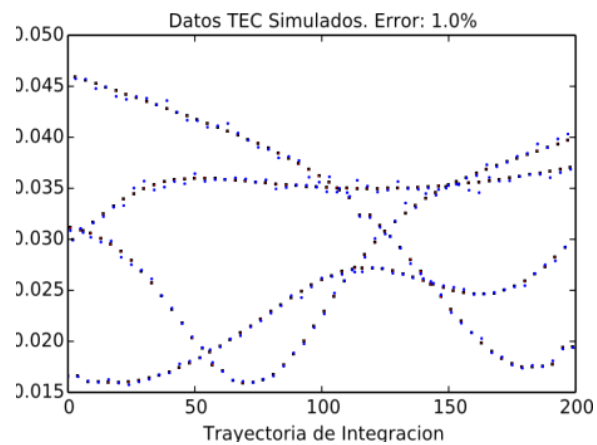

Figura 6: Datos TEC simulados (cuadrados). TEC + error (círculos).

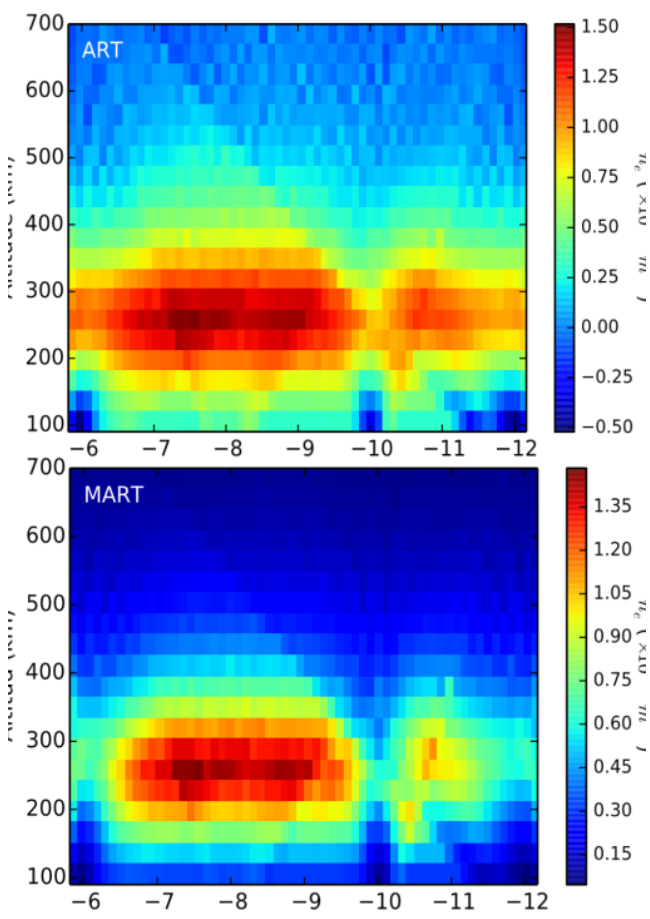

Figura 7: Reconstrucciones con ART (Arriba) y MART (Abajo).

El método seleccionado para la realización del TEC consiste en el cálculo de la diferencia de fases de dos ondas moduladas transmitidas desde satélites y recibidas en distintas estaciones terrestres que conforman una red de receptores. Debido a que cada medida individual contiene información incompleta, mientras más extensa sea la red de 

recepción, la reconstrucción se podrá obtener de manera más exacta; especialmente en el caso de altitudes bajas. [8]

La segunda consideración es que cada receptor de la red debe ser capaz de detectar el paso de múltiples satélites a diferentes frecuencias de manera simultánea. Este tipo de receptor es posible utilizando una radio definida por software o SDR, por sus siglas en inglés, lo que básicamente significa que la mayoría del procesamiento de señales se realiza con software en una computadora, en lugar de hardware de procesamiento de señal diseñado a medida. [8]

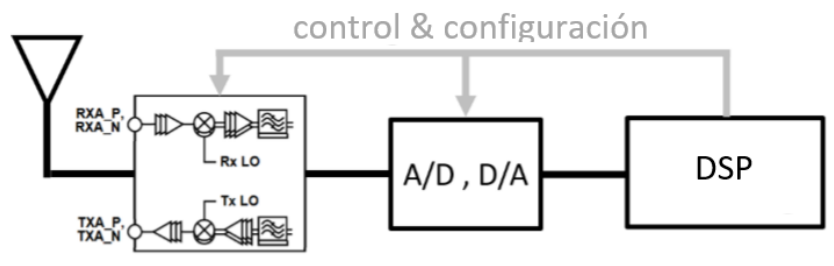

Figura 8: Diagrama de bloques SDR. Fuente: [10]

Un SDR está compuesto principalmente por una interfaz RF (Radio Frecuencia), conversores analógico-digital/digital-analógico (ADC/DAC), conversores digitales down/digital up (DDC/DUC) y un módulo de procesamiento digital de señales; tal como se muestra en la Figura 8.

Su gran flexibilidad y soporte de su comunidad la han convertido en una interesante herramienta en distintos ámbitos de la investigación, industria y educación. Para estudios en Radioastronomía y Astrofísica, el equipo más utilizado es el Universal Software Radio Peripheral (USRP) de la empresa National Instruments. Es así como en [8], Vierinen modernizó la red de receptores del Observatorio de Sodankyl, en Finlandia, basados en receptores de arquitectura SDR de 2 canales USRP. Este proyecto cuenta con una red de 4 USRP modelo N2x0 lo que representa un costo de 10,000 \$. En [9] M. Yamamoto presenta el desarrollo un sistema de recepción digital denominado GRBR (GNU Radio Beacon Receiver), basado en USRPs, cuyo objetivo es medir el TEC para el Research Institute for Sustainable Humanosphere, en Japón; reduciendo el costo a una relación de $1 / 100$, con respecto a otras opciones comerciales.

Pese a que la tecnología SDR ha permitido reducir los costos, actualmente, existe una gran variedad de equipos SDR de muy bajo costo con diferentes características. Con el fin de analizar si las alternativas basadas en SDR más económicos presentados en la Tabla 1, pueden ser adaptados a este proyecto, se pretende modelar un receptor digital.

Tabla 1: Cuadro comparativo SDR. Fuente: [10]

\begin{tabular}{|c|c|c|c|c|c|}
\hline & $\begin{array}{c}\text { LIME } \\
\text { SDR }\end{array}$ & $\begin{array}{c}\text { USRP } \\
\text { B210 }\end{array}$ & $\begin{array}{c}\text { BLADE } \\
\text { RF }\end{array}$ & $\begin{array}{c}\text { Hack } \\
\text { RF One }\end{array}$ & $\begin{array}{c}\text { RTL } \\
\text { SDR }\end{array}$ \\
\hline Price (\$) & 299 & 1200 & $300-450$ & $240-350$ & 20 \\
\hline $\begin{array}{c}\text { Frequency } \\
\text { (MHz) }\end{array}$ & $\begin{array}{c}0.1 \\
3800\end{array}$ & $\begin{array}{c}70 \\
6000\end{array}$ & $\begin{array}{c}300 \\
3800\end{array}$ & $\begin{array}{c}1 \\
6000\end{array}$ & $\begin{array}{c}0.5 \\
1750\end{array}$ \\
\hline TX/RX & $\begin{array}{c}6 \mathrm{RX} 4 \\
\mathrm{TX}\end{array}$ & $\begin{array}{c}2 \mathrm{RX} 2 \\
\mathrm{TX}\end{array}$ & $\begin{array}{c}\mathrm{RX} \\
\mathrm{TX}\end{array}$ & $\begin{array}{c}\mathrm{RX} \\
\mathrm{TX}\end{array}$ & $\mathrm{RX}$ \\
\hline $\begin{array}{c}\text { Resolution } \\
\text { (Bits) }\end{array}$ & 12 & 12 & 12 & 8 & 8 \\
\hline $\begin{array}{c}\text { Interface } \\
\text { USB }\end{array}$ & 3.0 & 3.0 & 3.0 & 2.0 & 2.0 \\
\hline $\begin{array}{c}\text { Sample Rate } \\
\text { (Msps) }\end{array}$ & 20 & 61.44 & 40 & 20 & 2.4 \\
\hline $\begin{array}{c}\text { Bandwidth } \\
\text { (MHz) }\end{array}$ & 64 & 56 & 28 & 20 & 3.2 \\
\hline
\end{tabular}

La simulación que se plantea cubriría dos etapas básicas de un receptor digital. La simulación cubre dos etapas básicas de un receptor: Un ADC cuya función es discretizar la señal entrante y, el DDC cuya función es demodular la señal entrante y llevarla a banda base. El objetivo del modelo consiste en calcular el error de fase inherente a los diferentes receptores, para poder compararlos, y concluir si son adecuados. Para este propósito, se pretende tener como entrada la resolución del ADC en bits.

Esta propuesta, abre la consideración de preparar prototipos económicos para radares pasivos como en [11]. Otra posible mejora es el uso de una tercera frecuencia para mejorar la robustez del experimento en [12].

\subsection{Determinación de los planos de reconstrucción}

La escala de variación de $n_{e}$ es de fundamental importancia para determinar el tamaño de la malla de reconstrucción, ya que estamos asumiendo que $n_{e}(t) \approx n e\left(t+\Delta t_{s a t}\right)$, donde $\Delta t_{s a t}$ es el tiempo que le tomaría al satélite cruzar el plano de reconstrucción. Para poder justificar esta aproximación, es necesario determinar el tipo de fenómenos que se pueden asumir estáticos en esta escala de tiempo. Las mediciones de $n_{e}$ del radar de dispersión incoherente del ROJ constituyen las estimaciones más precisas en espacio y tiempo de 
la densidad de electrones a las que podríamos acceder.
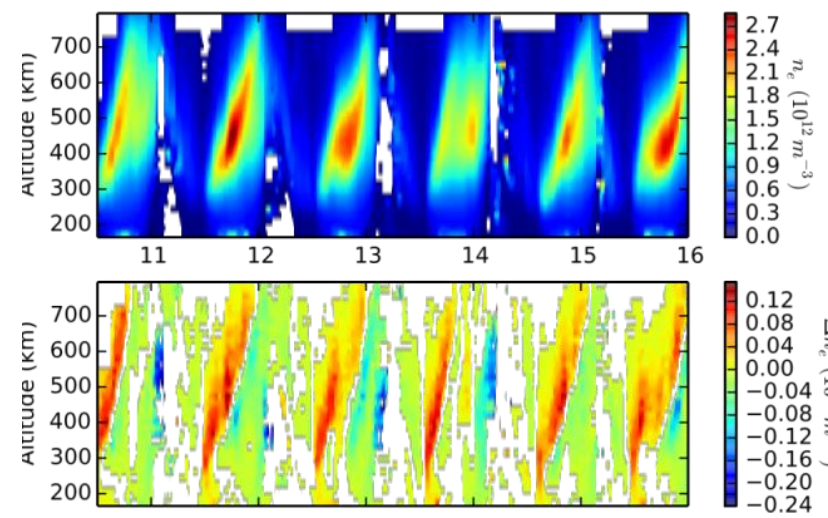

Figura 9: Mediciones del ISR $\left(n_{e}\right)$ cada $\approx 10 \mathrm{~min}$.

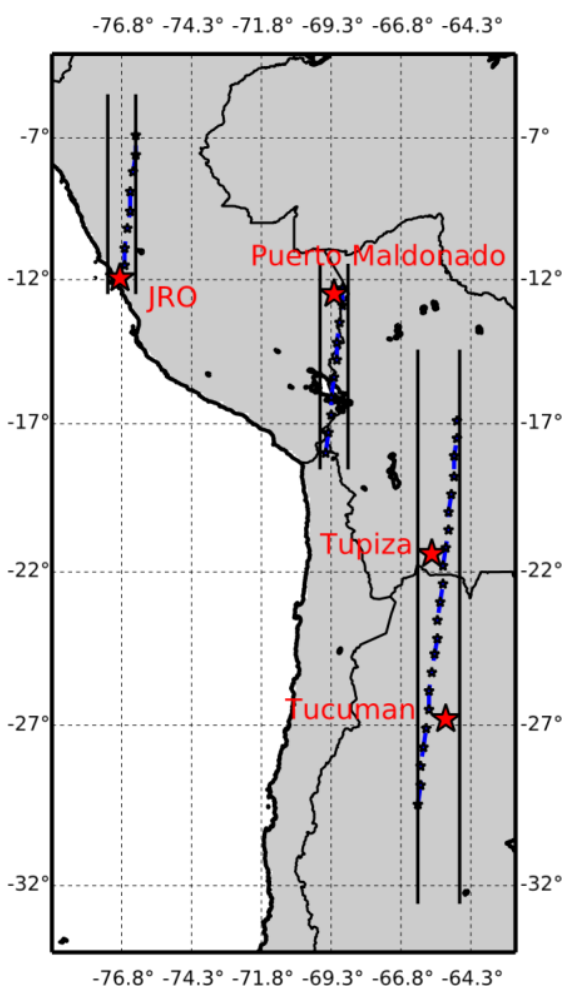

Figura 10: Planos de reconstrucción sobre ionosondas LISN.

La Figura 9 muestra los perfiles de densidad en la primera fila y la variación de estos perfiles a lo largo de 10 minutos en la segunda fila.

Podemos ver que la variación temporal de $n_{e}$ es del orden de magnitud que la precisión del radar $(\approx$ $0,1 n_{e}$ ), lo que sugiere que la variación de $n_{e}$ en 10 minutos, es suficientemente pequeña para que el radar no sea capaz de resolverla. Usando este criterio como primera aproximación, diremos que mientras $\Delta t_{\text {sat }} \ll 10 \mathrm{~min}$, podemos asumir que la ionósfera se encuentra estática al paso del satélite.

Tomando entonces trayectorias de 2 minutos del satélite CASSIOPE, podemos estimar la geometría de los posibles planos de reconstrucción; representados como líneas negras en la Figura 9. Estos planos contienen además al menos una ionosonda de la red LISN (señalada con la estrella roja), mientras que la trayectoria del satélite está representada con la línea azul.

\section{Conclusiones}

En este trabajo, se presentó algunos resultados preliminares de la estimación de perfiles de densidad de electrones de data simulada usando Multiplicative Algebraic Reconstruction Technique (MART) [5] y Simultaneous Iterative Reconstruction Technique (SIRT) [5].

Se modeló burbujas de plasma, zonas en las que la densidad de electrones decrece abruptamente, como las producidas por "Spread F" [7].

Trabajos futuros se enfocarán en mejorar el perfil ionosférico simulado y comparar nuestros resultados actuales con resultados obtenidos mediante regularización. Este trabajo será utilizado para diseñar un arreglo experimental para obtener data real.

\section{Referencias}

[1] Austen, J. R., S. J. Franke, and C. H. Liu (1988), lonospheric imaging using computerized tomography, Radio Sci., 23(3), 299-307.

[2] Bust, G. S., and C. N. Mitchell (2008), History, current state, and future directions of ionospheric imaging, Rev. Geophys., 46, RG1003.

[3] Das, S. K., and A. K. Shukla (2011), Two dimensional ionospheric tomography over the low latitude Indian region: An intercomparison of ART and MART algorithms, Radio Sci., 46, RS2005.

[4] Kudeki, Erhan., Applications of Radiowave Propagation. ECE 458, University of Illinois at Urbana-Champaign. 
[5] Kunitsyn, V. E. (2003), Ionospheric

Tomography, Springer-Verlag Berlin

Heidelberg, Germany.

[6] Hargreaves, J. K. The Solar-Terrestrial Environment: an Introduction to Geospace. Cambridge University Press, 1995.

[7] D.L. Hysell (2000), An overview and synthesis of plasma irregularities in equatorial spread F.Journal of Atmospheric and Solar-Terrestrial Physics, Volume 62, Issue 12,Pages 1037- 1056.

[8] Vierinen, J., et al. (2014) Beacon satellite receiver for ionospheric tomography. Radio Science 49.12, Pages 1141-1152

[9] M. Yamamoto (2008), Digital beacon receiver for ionospheric tec measurement developed with gnu radio, Earth, Planets and Space, vol. 60, no. 11, pp. e21-e24.
[10] H. Miyashiro, M. Medrano, J. Huarcaya y J. Lezama (2017), Software defined radio for hands-on communication theory. In Proceedings of the IEEE XXIV International Congress on Electronics, Electrical Engineering and Computing, Cusco, Perú.

[11] Javier Pérez Penedo (2015), Desarrollo de un receptor sdr de bajo coste para la medida del contenido de electrones de la ionosfera (TEC), Universidad de Vigo, 2015.

[12] P. A. Bernhardt y C. L. Siefring (2006), New satellite-based systems for ionospheric tomography and scintillation region imaging, Radio Science, vol. 41, no. 5, RS5S23.

Emails:melissa.medranoc@gmail.com, mmerino@upc.edu.pe, jpvelasquez@pucp.edu.pe, mar.inonan@gmail.com y elr96@cornell.edu 